respectively). However, among patients who received sunitinib, grade 3 or 4 treatment-related adverse events were higher among those in the older age group (55\% vs. $70 \%$ ).

Our results support an age-independent benefit-risk profile of nivolumab plus ipilimumab, including in elderly patients. Clinical data are lacking to assess any distinctive changes in the cellular immune response to checkpoint inhibitors as a function of age.

Robert J. Motzer, M.D.

Memorial Sloan Kettering Cancer Center

New York, NY

motzerr@mskcc.org
M. Brent McHenry, Ph.D.

Allen C. Chen, M.D.

Bristol-Myers Squibb

Princeton, NJ

Since publication of their article, the authors report no further potential conflict of interest.

1. Canaday DH, Parker KE, Aung H, Chen HE, Nunez-Medina $\mathrm{D}$, Burant CJ. Age-dependent changes in the expression of regulatory cell surface ligands in activated human T-cells. BMC Immunol 2013;14:45.

2. Wei SC, Levine JH, Cogdill AP, et al. Distinct cellular mechanisms underlie anti-CTLA-4 and anti-PD-1 checkpoint blockade. Cell 2017;170(6):1120-1133.e17.

DOI: $10.1056 /$ NEJMc1805988

\title{
Edoxaban for Cancer-Associated Venous Thromboembolism
}

TO THE EDITOR: Regarding the article by Raskob et al. (Feb. 15 issue) ${ }^{1}$ comparing the safety and efficacy of edoxaban with subcutaneous dalteparin in patients with cancer-associated venous thromboembolism, we believe that these results may affect the quality of life of patients with cancer. The inconvenience of the use of long-term subcutaneous low-molecular-weight heparin therapy might be avoided by the use of direct oral anticoagulant agents. The use of direct oral anticoagulants also reduces the cost of treatment for venous thromboembolism. However, low-molecular-weight heparin remains the standard of care for management of acute venous thromboembolism in patients with cancer. Guidelines currently recommend at least 6 months of therapy with low-molecular-weight heparin up front and do not have a preference for direct oral anticoagulants for patients who are not treated with lowmolecular-weight heparin. ${ }^{2}$

On the basis of the results of the current trial, some patients with cancer-associated venous thromboembolism should be switched to direct oral anticoagulants. Should we switch patients with rapidly progressive disease to direct oral anticoagulants, or should we consider this treatment only for patients with responsive or stable disease? Do the results of the current trial apply to all direct oral anticoagulants? Navigating this switch implies many challenges for clinician oncologists.

\author{
Maria G. Calabrò, M.D. \\ San Raffaele Hospital \\ Milan, Italy \\ Giuseppe Curigliano, M.D., Ph.D.
}

University of Milan

Milan, Italy

giuseppe.curigliano@ieo.it

No potential conflict of interest relevant to this letter was reported.

1. Raskob GE, van Es N, Verhamme P, et al. Edoxaban for the treatment of cancer-associated venous thromboembolism. N Engl J Med 2018;378:615-24.

2. Kearon C, Akl EA, Ornelas J, et al. Antithrombotic therapy for VTE disease: CHEST guideline and expert panel report. Chest 2016;149:315-52.

DOI: $10.1056 /$ NEJMc1806646

TO THE EDITOR: Regarding the article by Raskob et al. on edoxaban for cancer-associated venous thromboembolism, this trial showed the noninferiority of edoxaban to dalteparin with respect to the composite outcome of recurrent venous thromboembolism or major bleeding (the primary end point). Treatment with edoxaban or dalteparin was to be continued for at least 6 to 12 months in this trial. Although the treatment period defined by the protocol was longer than that in previous studies, ${ }^{1}$ events of recurrent venous thromboembolism were observed throughout the treatment period. According to treatment guidelines for venous thromboembolism in patients with cancer, ${ }^{2}$ anticoagulant therapy with 
low-molecular-weight heparin is recommended for at least 6 months. However, there is no consensus about the maximum treatment period. Edoxaban is a novel oral agent and will be regarded as a new treatment option for cancerrelated thromboembolism, but a new question has now arisen: for how many months should we continue to give edoxaban?

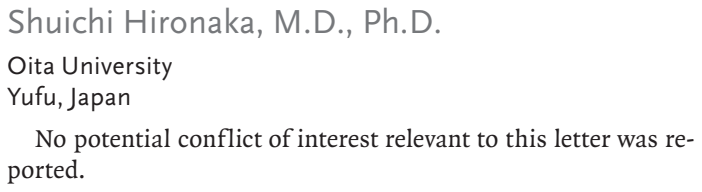

No potential conflict of interest relevant to this letter was reported.

1. Posch F, Königsbrügge O, Zielinski C, Pabinger I, Ay C. Treatment of venous thromboembolism in patients with cancer: a network meta-analysis comparing efficacy and safety of anticoagulants. Thromb Res 2015;136:582-9.

2. Lyman GH, Bohlke K, Falanga A. Venous thromboembolism prophylaxis and treatment in patients with cancer: American Society of Clinical Oncology clinical practice guideline update. J Oncol Pract 2015;11:e442-e444.

DOI: 10.1056/NEJMc1806646

TO THE EDITOR: Raskob et al. report a higher rate of major gastrointestinal bleeding with edoxaban than with dalteparin among patients with cancer treated for venous thromboembolism. The increased risk of gastrointestinal bleeding with direct oral anticoagulants remains poorly understood. Unlike antithrombin-dependent factor Xa inhibitors, direct factor Xa inhibitors inactivate both free and prothrombinase-bound factor Xa, further decreasing local thrombin generation and activation of platelets and endothelial cells through protease-activated receptors. Concordantly, edoxaban suppresses tissue factor-induced platelet aggregation in vitro $^{1}$ and was shown to fully inhibit arterial thrombosis in a rat model. ${ }^{2}$ In addition, direct factor Xa inhibitors modulate platelet glycoprotein VI shedding and the urinary excretion of thromboxane $\mathrm{B}_{2}$, which indicate impaired platelet activation and thromboxane biosynthesis. ${ }^{3,4}$

In patients with cancer, edoxaban-mediated antiplatelet effects might contribute to gastrointestinal bleeding as a result of an impaired ability of platelets to form a plug at the endothelial surface, particularly at sites of highly vascular and friable tumor, which are known to bleed persistently. Thus, in patients with such gastrointestinal tumors, low-molecular-weight heparin remains a better option than direct oral anticoagulants.
Corinne Frere, M.D., Ph.D.

Monique Dufilho, Ph.D.

Sorbonne Universités

Paris, France

corinne.frere@aphp.fr

No potential conflict of interest relevant to this letter was reported.

1. Honda Y, Kamisato C, Morishima Y. Edoxaban, a direct factor Xa inhibitor, suppresses tissue-factor induced human platelet aggregation and clot-bound factor Xa in vitro: comparison with an antithrombin-dependent factor Xa inhibitor, fondaparinux. Thromb Res 2016;141:17-21.

2. Honda $Y$, Kamisato C, Morishima Y. Prevention of arterial thrombosis by edoxaban, an oral factor Xa inhibitor in rats: monotherapy and in combination with antiplatelet agents. Eur J Pharmacol 2016;786:246-52.

3. Al-Tamimi M, Grigoriadis G, Tran H, et al. Coagulationinduced shedding of platelet glycoprotein VI mediated by factor Xa. Blood 2011;117:3912-20.

4. Pignatelli P, Pastori D, Bartimoccia S, et al. Anti Xa oral anticoagulants inhibit in vivo platelet activation by modulating glycoprotein VI shedding. Pharmacol Res 2016;113:484-9.

DOI: $10.1056 /$ NEJMc1806646

TO THE EDITOR: Treatment of patients with cancer with heparin and low-molecular-weight heparin is associated with increased survival. ${ }^{1}$ The report by Raskob et al. shows a trend toward a higher survival rate among patients treated with low-molecular-weight heparin than among those treated with edoxaban. Heparin and low-molecularweight heparin have anticancer properties in animal models. ${ }^{2}$ We have shown that heparanase, an enzyme secreted from most cancer cells that degrades heparan sulfate in the extracellular matrix, is inhibited by heparin and low-molecularweight heparin through competitive inhibition. ${ }^{3}$ Heparin is a natural product extracted from bovine lungs or pig intestine. Low-molecular-weight heparin is a semisynthetic molecule. We have shown that transgenic mice overexpressing heparanase have lower baseline activated partialthromboplastin time values than control mice. ${ }^{3}$

Patients with cancer with high serum levels of heparanase have resistance to standard doses of low-molecular-weight heparin. ${ }^{4}$ Hypercoagulation in patients with cancer seems to be related to molecules secreted from tumors such as heparanase, which degrades endogenous heparin. ${ }^{3,4}$ Treatment of patients with cancer with heparin and low-molecular-weight heparin is indicated and should be coupled with close monitoring of anti-factor Xa activity and dose adjustment to achieve therapeutic levels. ${ }^{4}$ 
Nicola J. Nasser, M.D., Ph.D.

Montefiore Medical Center

Bronx, New York

nnasser@montefiore.org

No potential conflict of interest relevant to this letter was reported.

1. Altinbas M, Coskun HS, Er O, et al. A randomized clinical trial of combination chemotherapy with and without low-molecular-weight heparin in small cell lung cancer. J Thromb Haemost 2004;2:1266-71.

2. Niers TM, Klerk CP, DiNisio M, et al. Mechanisms of heparin induced anti-cancer activity in experimental cancer models. Crit Rev Oncol Hematol 2007;61:195-207.

3. Nasser NJ, Sarig G, Brenner B, et al. Heparanase neutralizes the anticoagulation properties of heparin and low-molecularweight heparin. J Thromb Haemost 2006;4:560-5.

4. Nasser NJ, Na'amad M, Weinberg I, Gabizon AA. Pharmacokinetics of low molecular weight heparin in patients with malignant tumors. Anticancer Drugs 2015;26:106-11.

DOI: $10.1056 /$ NEJMc1806646

THE AUTHORS REPLY: We agree with Calabrò and Curigliano that oral therapy with edoxaban is likely to improve the quality of life of patients with cancer-associated venous thromboembolism. We disagree, however, that low-molecular-weight heparin remains the standard of care for venous thromboembolism in patients with cancer. A possible direction derived from our results is that, after 5 days of low-molecular-weight heparin, edoxaban can replace low-molecular-weight heparin for most patients with active cancer, including those with advanced disease. The guidelines ${ }^{1}$ were written and published before our results were available. Our results with edoxaban may not be translatable to other oral anticoagulants, since many anticancer therapies affect the cytochrome P450 system, and the minimal metabolism of edoxaban by this system distinguishes it from other oral factor Xa inhibitors. ${ }^{2}$

In response to Hironaka: evidence-based guidelines recommend extended anticoagulant therapy (no scheduled stop date) for a duration of more than 3 months in patients with venous thromboembolism and cancer who do not have a high risk of bleeding. ${ }^{1}$ Oral edoxaban facilitates extended anticoagulant therapy and eliminates the need for months of subcutaneous injections of lowmolecular-weight heparin, providing an opportunity for enhanced patient adherence. Our results show the effectiveness and safety of edoxaban treatment for up to 1 year. We speculate that treatment should probably be continued until the cancer is resolved because these patients have a high risk of recurrent venous thromboembolism.

Frere and Dufilho raise interesting speculations on the mechanism of gastrointestinal bleeding that is associated with edoxaban in patients with gastrointestinal tumors. We disagree that low-molecular-weight heparin remains a better option for all patients with gastrointestinal tumors. Some patients may choose the convenience of oral edoxaban despite an increased risk of gastrointestinal bleeding, ${ }^{3}$ especially since we found that the frequency of severe major bleeding was similar with edoxaban and dalteparin. Stopping injections of low-molecular-weight heparin early (e.g., after 1 to 2 months), with the attendant increased risk of recurrent venous thromboembolism, could offset the lower risk of bleeding. These trade-offs should be discussed with patients and the patient's preference considered.

The trial cited by Nasser is in the context of thromboembolism prevention during chemotherapy and is not relevant to the treatment of patients with established thromboembolism. The clinical trial data on a survival benefit of lowmolecular-weight heparin in patients with cancer without established thrombosis are conflicting. ${ }^{4}$ We do not agree that monitoring of anti-factor Xa activity is indicated in patients with cancer treated with low-molecular-weight heparin owing to the lack of evidence that this improves the patient's outcome. Edoxaban does not require anticoagulant monitoring and provides an effective, convenient alternative to low-molecularweight heparin for most patients with cancerassociated venous thromboembolism.

\section{Gary E. Raskob, Ph.D.}

University of Oklahoma Health Sciences Center

Oklahoma City, OK

gary-raskob@ouhsc.edu

Harry R. Büller, M.D., Ph.D.

Academic Medical Center

Amsterdam, the Netherlands

Annelise Segers, M.D.

ITREAS Clinical Research

Amsterdam, the Netherlands

Since publication of their article, the authors report no further potential conflict of interest.

1. Kearon C, Akl EA, Ornelas J, et al. Antithrombotic therapy for VTE disease: CHEST guideline and expert panel report. Chest 2016;149:315-52.

2. Parasrampuria DA, Truitt KE. Pharmacokinetics and pharmacodynamics of Edoxaban, a non-vitamin $\mathrm{K}$ antagonist oral 
anticoagulant that inhibits clotting factor Xa. Clin Pharmacokinet 2016;55:641-55.

3. Hirsh J, Ginsberg JS. Edoxaban for the treatment of venous thromboembolism in patients with cancer. N Engl J Med 2018; 378:673-4.
4. van Doormaal FF, Di Nisio M, Otten HM, Richel DJ, Prins M, Buller HR. Randomized trial of the effect of the low molecular weight heparin nadroparin on survival in patients with cancer. J Clin Oncol 2011;29:2071-6.

DOI: 10.1056/NEJMc1806646

\section{Primary Sjögren's Syndrome}

TO THE EDITOR: Mariette and Criswell (March 18 issue $)^{1}$ rightly point out that chronic pain and fatigue are hallmarks of primary Sjögren's syndrome, but they do not address the neurologic contributors to these symptoms. Although the central nervous system is indeed rarely affected in patients with primary Sjögren's syndrome ( $<5 \%$ of patients), the prevalence of peripheral neuropathy ranges between 16 and $25 \%,{ }^{2,3}$ which is higher than the $6 \%$ prevalence reported by Mariette and Criswell. Small-fiber polyneuropathies account for at least $30 \%$ of these cases. ${ }^{2}$ Large-fiber involvement causes ataxia, sensory loss, or motor deficit, whereas small-fiber involvement contributes to pain in particular, regardless of whether symptoms are length-dependent (distal polyneuropathy) or not (ganglionopathy). Smallfiber denervation of the heart and microvessels may contribute to fatigue and exertional intolerance, and small-fiber denervation of lachrymal and salivary glands may contribute to the sicca syndrome.

Given these prevalent neurologic symptoms and the absence of antibodies against Sjögren's syndrome-related antigen A (anti-SSA) in 60\% of the patients with primary Sjögren's syndrome and small-fiber polyneuropathy, ${ }^{2}$ salivary-gland biopsy is an important consideration in patients with otherwise unexplained small-fiber polyneuropathy. Questionnaires and laboratory tests for smallfiber polyneuropathy, including skin biopsy and autonomic and neurophysiological tests, ${ }^{4}$ aid in the diagnosis. The results will influence decisions regarding treatments for symptoms (e.g., tricyclic antidepressants, gabapentin, pregabalin) and immunotherapies (e.g., immune globulin infusions). ${ }^{5}$ Thus, neurologic considerations are integral to the diagnosis and treatment of primary Sjögren's syndrome.

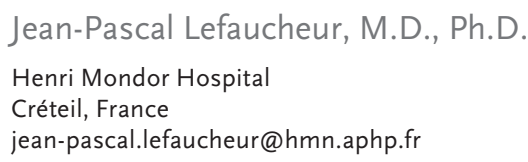

\author{
Damien Sène, M.D., Ph.D. \\ Lariboisière Hospital \\ Paris, France \\ Anne Louise Oaklander, M.D., Ph.D. \\ Massachusetts General Hospital \\ Boston, MA
}

No potential conflict of interest relevant to this letter was reported.

1. Mariette X, Criswell LA. Primary Sjögren's syndrome. N Engl J Med 2018;378:931-9.

2. Sène $\mathrm{D}$, Jallouli $M$, Lefaucheur JP, et al. Peripheral neuropathies associated with primary Sjögren syndrome: immunologic profiles of nonataxic sensory neuropathy and sensorimotor neuropathy. Medicine (Baltimore) 2011;90:133-8.

3. Carvajal Alegria G, Guellec D, Mariette X, et al. Epidemiology of neurological manifestations in Sjögren's syndrome: data from the French ASSESS cohort. RMD Open 2016;2:e000179.

4. Treister R, Lodahl M, Lang M, Tworoger SS, Sawilowsky S, Oaklander AL. Initial development and validation of a patientreported symptom survey for small-fiber polyneuropathy. J Pain 2017;18:556-63.

5. Liu X, Treister R, Lang M, Oaklander AL. IVIg for apparently autoimmune small-fiber polyneuropathy: first analysis of efficacy and safety. Ther Adv Neurol Disord 2018;11:17562856177444484.

DOI: 10.1056/NEJMc1804598

TO THE EDITOR: Mariette and Criswell identify the systemic complications of primary Sjögren's syndrome, but they do not mention the increased burden of cardiovascular disease, as has been described in other systemic autoimmune diseases such as systemic lupus erythematosus and rheumatoid arthritis. ${ }^{1,2}$ Women with primary Sjögren's syndrome are more likely to have myocardial infarction and cerebrovascular events than are healthy women of similar age. ${ }^{3}$ Hypertension and hypercholesterolemia were more prevalent among these patients with primary Sjögren's syndrome, but other traditional risk factors such as smoking, obesity, and diabetes mellitus were not. ${ }^{3}$ Underlying systemic inflammation has been proposed to contribute to this increased risk.

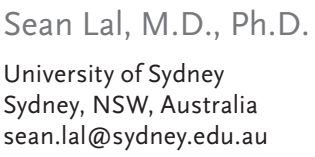

